\title{
Demonstration of the APRON Processor Array Simulation Software
}

\author{
David R. W. Barr and Piotr Dudek
}

School of Electrical \& Electronic Engineering, The University of Manchester e-mail: d.barr@postgrad.manchester.ac.uk; p.dudek@manchester.ac.uk

\begin{abstract}
A software tool for the development, prototyping and emulation of cellular processor array hardware is presented. APRON provides a 'virtual processor array' that operates at high speed, that can be extended to form vision systems, multi-layer neural networks, cellular neural networks and neuromorphic arrays.
\end{abstract}

\section{SUMMARY}

Cellular Processor Arrays (CPAs) like those in [1,2] implement a high-density grid of computationally simple

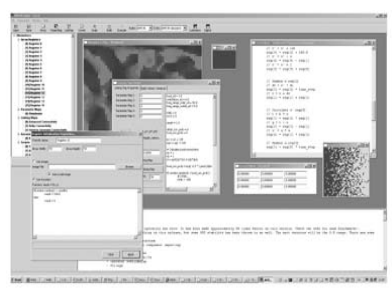

(a)

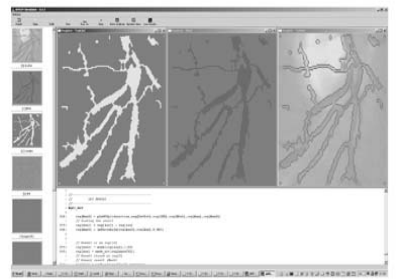

(c)

(e)

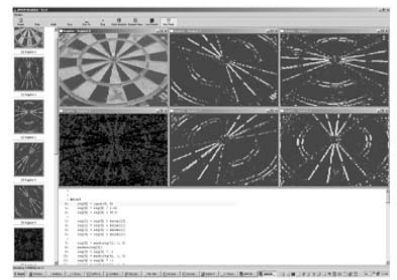

(g)

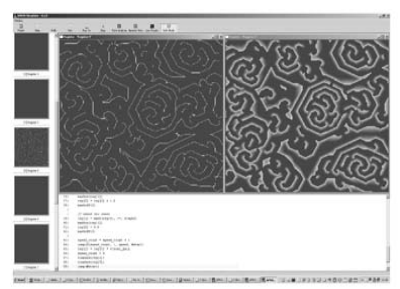

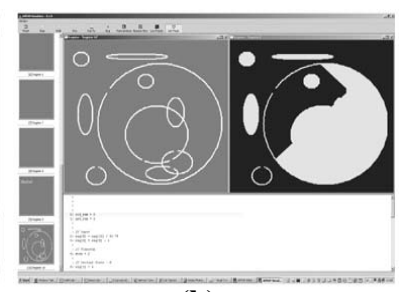

(b)

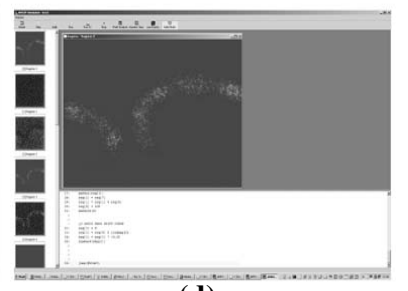

(d)

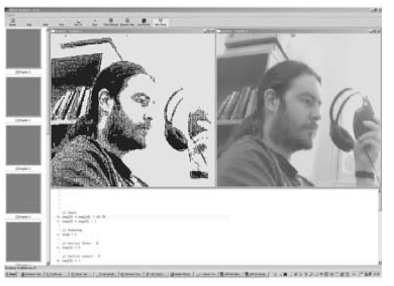

(f)

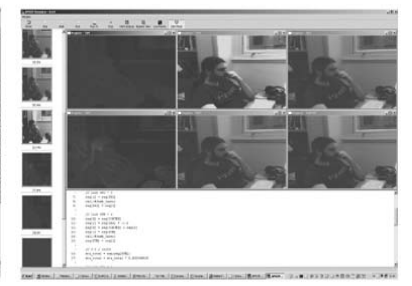

(h)
Figure 1 - Various algorithms being simulated within the APRON environment (See text for details) processors, with local memories, and neighbourhood communication, operating under the Single Instruction Multiple Data (SIMD) paradigm. This arrangement has proven to be particularly suited to pixel-parallel image processing, cellular neural networks (CNNs), multi-layer neural networks, neuromorphic arrays, and computational neuroscience models. This suitability is largely due to the elimination of any memory access bottlenecks, traditionally associated with serial implementations of the same applications, and specifically designed functional components within the processors. The field of CPA related research has been growing steadily, therefore so has the development of new hardware.

We have developed a software system that can be used at all stages in the lifecycle of a CPA device. APRON (Array Processing enviRONment) is a flexible, processor array design and simulation tool [3], which has been optimised for high speed and accuracy on a standard desktop computer. This demonstration will show the APRON system performing a variety of algorithms, with a bias towards vision system emulation. Through the use of a webcam, images are captured and processed fast-enough to result in an interactive simulation. Figure 1 shows the APRON software executing several algorithms: a) The APRON IDE, b) Hole-Filling using the CNN paradigm, c) Retina Vessel Extraction using Pixel Level Snakes [4], d) biologically plausible excitable medium using Izhikevich neurons and a probabilistic Gaussian connectivity strategy, e) Greenberg-Hastings cellular Automata, f) Half-toning using CNN in real-time, g) Salient feature extraction in real-time, h) Multi-layer model of Intrinsic Basal Ganglia [5]. We also show how the software can be used as a hardware prototyping tool and CPA development environment, implementing as an example the ASPA [1] architecture and its instruction set.

\section{REFERENCES}

[1] A.Lopich and P.Dudek, "Global operations on SIMD cellular processor arrays: towards functional asynchronism", International Workshop on Computer Architectures for Machine Perception and Sensing, CAMPS 2006

[2] P.Dudek, "Implementation of SIMD Vision Chip with $128 \times 128$ Array of Analogue Processing Elements", ISCAS 2005, Kobe, pp.5806-5809

[3] D. R. W. Barr and P. Dudek, "A Cellular Processor Array Simulation and Hardware Prototyping Tool", IEEE Workshop on Cellular Neural Networks and their Applications, CNNA 2008 (SUBMITTED)

[4] C.Alonso-Montes, P.Dudek, D.L.Vilarino and M.G.Penedo, "On Chip implementation of a Pixel-Parallel Approach for Retinal Vessel Tree Extraction", ECCTD 2007, pp.511-514, Seville, Spain, August 2007

[5] D.R.W. Barr, P. Dudek, J. Chambers and K. Gurney, "Implementation of multi-layer leaky integrator networks on a cellular processor array", IJCNN 2007, Orlando, Florida 